(Invited paper for proceedings of Physics Education Research Conference, 2001)

\title{
Inductive Influence of Related Quantitative and Conceptual Problems
}

\author{
Phillip Dukes and David E. Pritchard \\ MIT, Cambridge, MA 02139-4307
}

\begin{abstract}
Physics education research shows that conceptual understanding is not necessary for students to do well on standard quantitative problems in introductory physics. This study addresses a related question: is conceptual understanding sufficient or helpful for students to do well in quantitative problems?
\end{abstract}

We approached this question by splitting a class of $\sim 100$ students into two equal groups, A and B, and giving the A group a conceptual problem prior to giving a related quantitative problem to both groups. If the A group experiences less difficulty with the quantitative problem than the B group, we say that the conceptual problem has positive "inductive influence" on the quantitative problem.

By administering the same conceptual problem to the B group after they do the quantitative one, our methodology also addresses the reverse problem "How much conceptual knowledge does a student learn from working through a quantitative problem?"

Surprisingly (to us anyway), our results show little inductive influence of the conceptual problem on the quantitative one, but suggest that working the quantitative problem first may help students with the subsequent conceptual one.

\section{CyberTutor measures difficulty}

The current study was undertaken during the Spring 2001 term of the required Introductory Newtonian mechanics with calculus course at MIT. Problem pairs were administered using CyberTutor, a web-based tutorial program. CyberTutor behaves like a Socratic tutor, offering students help upon request in the form of hints and simpler subproblems, spontaneous warnings and helpful suggestions when wrong answers are given, and the correct solution if the student exhausts the hints and requests it. Typically about $90 \%$ of the students work their way through to the solution, the remaining $10 \%$ requesting the solution. CyberTutor keeps a record of the number of hints requested (h) right (r) and wrong (w) answers submitted, and correct solutions (s) requested. These request for help are weighted and used to determine a measure for the difficulty a student experiences with a problem according to

Difficulty $=\log ((1+r+w+3 h+9 s) /(1+r))$.

The difficulty is 0 if only right answers are given and rises to around 3 for students requesting all the hints and solutions with no right answers. Values around 1 appear to be educationally optimum.

The two class groups were hand selected so that they had equal numbers of students taking a more advanced math course (than second semester calculus on multi-variable functions), equal numbers 
taking the first semester calculus course (most for the second time), and found the problems on the first two assignments on CyberTutor (http://cybertutor.mit.edu) of equal difficulty. The difference in difficulty rating stayed remarkably constant over the term, never differing by more than $4 \%$.

\section{An Example}

Here is a pair of problems concerning two body collisions in one dimension. Problem 1C is largely conceptual and 1Q is quantitative.

Problem 1C [conceptual]. Bullet Embedding in a Block

A bullet of mass $\mathrm{m}$ is fired horizontally with speed $\mathrm{v}_{0}$, aimed at a block of mass $M$ resting on a frictionless table. It hits the block, and becomes completely embedded. The block and bullet then move at speed $v_{f}$.

Part A. Which of the following best describes this collision?

- perfectly elastic.

- partially inelastic.

- perfectly inelastic.

- none of the above.

Part B. Which of the following quantities, if any, are conserved during this collision?

- kinetic energy.

- kinetic energy and momentum.

- momentum.

- none of the above.

Part C. What is the speed of the block+bullet system after the collision in terms of $\mathrm{v}_{0}, \mathrm{~m}$, and $\mathrm{M}$ ?

Problem 1Q [quantitative/numerical]. $\underline{\text { One-Dimensional Inelastic Collision }}$
Block 1, of mass $100 \mathrm{~g}$, moves along a frictionless airtrack with speed $0.2 \mathrm{~m} / \mathrm{s}$. It collides with block 2 , which was initially at rest. Block 2 has mass $200 \mathrm{~g}$. The blocks stick together and move as one after the collision.

Part A Find the total initial momentum $\mathrm{p}$ of the two-block system in units of $\mathrm{kg}$ $\mathrm{m} / \mathrm{s}$.

Part B. Find the final velocity $\mathrm{v}$ of the two blocks in $\mathrm{m} / \mathrm{s}$.

Part C. What is the change in the system's kinetic energy due to the collision? Express your answer in Joules.

A total of 78 students worked both problems. The 31 students who took problem 1C first followed by problem 1Q are group A1; the remaining 47 students in group B1, took the problems in reverse order. The average measured difficulty, D, for each group of students for each problem and the change in the difficulty after experience with the other problem is listed in Table 1.

\begin{tabular}{|c|c|c|}
\hline $\begin{array}{c}\text { Group } \\
\text { (Sample) }\end{array}$ & $\begin{array}{c}\text { Problem 1C } \\
\text { (Conceptual) }\end{array}$ & $\begin{array}{c}\text { Problem 1Q } \\
\text { (Quantitative) }\end{array}$ \\
\hline $\mathrm{A} 1$ & $($ First) & $($ Second) \\
$(31)$ & $\mathrm{D}=.2635$ & $\mathrm{D}=.7142$ \\
\hline $\mathrm{B} 1$ & $($ Second) & $($ First $)$ \\
$(47)$ & $\mathrm{D}=.1477$ & $\mathrm{D}=.6146$ \\
\hline \hline $\mathrm{D}^{\mathrm{S}}-\mathrm{D}^{\mathrm{F}}$ & $\Delta \mathrm{D}=-.1158$ & $\Delta \mathrm{D}=.0996$ \\
\hline \hline $\mathrm{P}$-value & $\mathrm{P}=.046$ & $\mathrm{P}=.56$ \\
\hline
\end{tabular}

Table 1. Measured difficulties for problems $1 \mathrm{C}$ and $1 \mathrm{Q}$ depending on the order in which they were taken.

The P-value in the table is the doublesided found using Student's t-test. ${ }^{1}$ This $P$ value is the probability that the observed discrepancy between the two sample means is due to chance. The more 
conservative two-sided $\mathrm{P}$ value is appropriate when sign of the effect is not known a priori.

Under the criteria that $\mathrm{P}<0.05$ is significant, we find that the students' experience with the quantitative problem was a significant help with their performance on the conceptual problem (it decreased the difficulty by $\Delta \mathrm{D}=$ .1158). The decrease in student difficulty with the conceptual problem was the result of an average of $45 \%$ fewer wrong answers, $\mathrm{w}, 71 \%$ fewer requests for hints, $\mathrm{h}$, and $0 \%$ fewer requests for correct solutions, s, see Table 2.

\begin{tabular}{|c|c|c|c|}
\hline Event & $\mathrm{W}$ & $\mathrm{h}$ & $\mathrm{S}$ \\
\hline$\%$ Decrease & $45 \%$ & $71 \%$ & $0 \%$ \\
\hline
\end{tabular}

Table 2. Percent decrease of CyberTutor help events for conceptual problem 1Q as a result of taking quantitative problem $1 \mathrm{C}$ first.

If anything, students found the quantitative problem 1Q of greater difficulty $(\Delta \mathrm{D}=+.0996)$ if they had first worked through the conceptual problem $1 \mathrm{C}$.

\section{Other Conceptual/Quantitative Pairs}

The results from other conceptual and quantitative pairs of problems are not so significant as the previous example. Due to growing (over the term) student selfselection of the order in which the problems were taken, a large percentage of the class ended up taking typical pairs is one sequence with very few taking them in the reverse order. One such example involved two problems dealing with contact forces and friction.

Prob. 2C [Conceptual] Contact Forces Explained
This is a conceptual problem, which begins by describing the general nature of contact forces and friction, then poses three multiple-choice questions for the student to answer.

Prob. 2Q. [Quantitative] Friction of a man on a drawbridge This problem describes a mass sliding with friction on an incline and asks the student for both quantitative and analytic responses.

The results for this pair of problems are given in Table 3.

\begin{tabular}{|c|c|c|}
\hline $\begin{array}{c}\text { Group } \\
\text { (Sample) }\end{array}$ & $\begin{array}{c}\text { Problem 2C } \\
\text { (Conceptual) }\end{array}$ & $\begin{array}{c}\text { Problem 2Q } \\
\text { (Quantitative) }\end{array}$ \\
\hline $\mathrm{A} 2$ & $\begin{array}{c}\text { (First) } \\
(70)\end{array}$ & $\begin{array}{c}\text { (Second) } \\
1.022\end{array}$ \\
\hline $\mathrm{B} 2$ & $\begin{array}{c}\text { (Second) } \\
(17)\end{array}$ & $\begin{array}{c}\text { (First) } \\
4036\end{array}$ \\
1.029 \\
\hline \hline $\mathrm{D}^{\mathrm{S}}-\mathrm{D}^{\mathrm{F}}$ & $\Delta \mathrm{D}=-.135$ & $\Delta \mathrm{D}=-.007$ \\
\hline \hline P-value & $\mathrm{P}=.0843$ & $\mathrm{P}=.9717$ \\
\hline
\end{tabular}

Table 3. Measured difficulties for problems $2 \mathrm{C}$ and $2 \mathrm{Q}$ depending on the order in which they were taken.

A third conceptual - quantitative comparison was performed in twodimensional kinematics. A conceptual problem involving the direction of acceleration of a car going around an irregular racetrack at various speeds was placed before and after a pair of quantitative problems: taking the derivative of a time-dependent $x-y$ position, and a similar problem involving circular motion. All measured inductive influences were small and statistically insignificant in this example.

\section{Conclusions}


Two findings emerge from the present small study:

1) Comparing three conceptual problems with four quantitative ones, we found no evidence of inductive influence of a previously administered conceptual problem on a subsequent quantitative problem on the same topic. In contrast to the results here, similar studies of the inductive influence of a numerical problem and a similar quantitative one, as well as the inductive influence of two closely related analytic problems show frequent reductions of difficulty over $50 \%$ with corresponding $\mathrm{P}$ values below 0.01 .

2) Statistically significant examples of inductive influence found were that the quantitative problem seemed able to help the students with a subsequent conceptual one. This may be an example of the general trend ${ }^{2}$ in our studies: that harder problems more often significantly reduced the difficulty of subsequent easier problems.

We would like to teach students to be physicists: to reason starting from a few key concepts through the related mathematics to a quantitative solution. However, this does not imply that the abstract concepts should be taught first. Indeed, this study suggests that only the reverse order shows positive inductive influence between concepts and quantitative problems. Perhaps students learn general concepts from particular examples, as Laurillard suggests in Rethinking University Teaching ${ }^{3}$ and Redish $^{4}$ has independely suggested that "Mental models must be built. People learn better by doing than by watching something being done" If this were correct, integrating concepts into the solution of quantitative problems would be educationally fruitful. Our data suggest that this may be the preferred approach.

This work was supported by NSF grant PHY-9988732. We are grateful to Alex Pritchard for developing CyberTutor and its ability to perform class splits, and to David Kokorowski for writing scripts to correct for students' doing problems out of the assigned order.

1 L. Horowitz, Elements of Statistics for Psychology and Education (McGrawHill, New York NY, 1974)

${ }^{2} \mathrm{~J}$. Belcher. Trends in Science

Education. ([on-line] url:

http://web.mit.edu/jbelcher/www/trends. html)

${ }^{3}$ Diana Laurillard Rethinking University Teaching - a framework for the effective use of educational technology

(Routledge, New York, NY,1993)

${ }^{4}$ E. Redish The Implications of Cognitive Studies for Teaching Physic. (The American Journal of Physics 62, 796-803, 1994) 